\title{
Factors Associated with Sustained Exergaming: Longitudinal Investigation
}

Erin Kathleen O'Loughlin ${ }^{1,2}$, MA; Tracie A Barnett ${ }^{1,3}, \mathrm{PhD}$; Jennifer J McGrath ${ }^{1}, \mathrm{PhD}$; Mia Consalvo ${ }^{1}, \mathrm{PhD}$; Lisa Kakinami ${ }^{1}, \mathrm{PhD}$

${ }^{1}$ Concordia University, Montreal, QC, Canada
${ }^{2}$ Centre de Recherche du Centre Hospitalier de l'Université de Montréal, Montreal, QC, Canada
${ }^{3}$ Le Centre L'Institut National de la Recherche Scientifique-Institut Armand-Frappier, Laval, QC, Canada

Corresponding Author:

Erin Kathleen O'Loughlin, MA

Concordia University

1455 De Maisonneuve Blvd

Montreal, QC, H3G 1M8

Canada

Phone: 15148908000

Email: erin oloughlin@hotmail.com

\section{Abstract}

Background: Exergaming is technology-driven physical activity (PA) which, unlike traditional video game play, requires that participants be physically active to play the game. Exergaming may have potential to increase PA and decrease sedentary behavior in youth, but little is known about sustained exergaming.

Objective: The objectives of this study were to describe the frequency, correlates, and predictors of sustained exergaming.

Methods: Data were available in AdoQuest (2005-11), a longitudinal investigation of 1843 grade 5 students in Montréal, Canada. This analysis used data from grade 9 (2008-09) and 11 (2010-11). Participants at Time 1 (T1; mean age 14 years, SD 0.8 ) who reported past-week exergaming $(n=186,19.1 \%$ of AdoQuest sample) completed mailed self-report questionnaires at Time 2 (T2; mean age 16 years, SD 0.8). Independent sociodemographic, psychological, and behavioral correlates (from T2)/predictors (from $\mathrm{T} 1$ or earlier) were identified using multivariable logistic regression.

Results: Of 186 exergamers at T1, $81(44 \%)$ reported exergaming at T2. Being female and having higher introjected regulation (ie, a type of PA motivation indicative of internalizing PA as a behavior) were independent correlates. None of the predictors investigated were associated with sustained exergaming.

Conclusions: Almost half of grade 9 exergamers sustained exergaming for 2 years. Exergaming may be a viable approach to help adolescents engage in and sustain PA during adolescence. Sex and PA motivation may be important in the sustainability of exergaming.

(JMIR Serious Games 2019;7(3):e13335) doi: 10.2196/13335

\section{KEYWORDS}

video games; physical activity; adolescents

\section{Introduction}

\section{Background}

Only $8 \%$ of Canadian youth meet current physical activity (PA) recommendations [1] and they spend up to 9 hours sitting daily, often in screen-time pursuits $[1,2]$. Youth who do not meet PA recommendations are unlikely to benefit from the positive effects of PA on health [3,4], and sedentary youth may experience a wide range of negative physical and mental health issues [5]. Effective programs and public health policy are needed to address these issues because co-occurrence of low PA and high sedentary time contributes to childhood obesity and its numerous, deleterious health sequelae [6-9]. Despite possible negative impacts on health, screens are an indisputable reality in today's increasingly high-tech world. Underscoring its popularity, $83 \%$ of US youth aged 8 to 18 years have a traditional video game console at home, and many of these consoles having add-on capacity for exergaming (ie, video games that are also a form of exercise) equipment. In addition, 
approximately $50 \%$ of adolescents own smartphones, which can download apps for mobile exergaming [10]. Many smartphone apps are free or low-cost, making exergaming and augmented reality (ie, a real-world view of the environment which is "augmented" by computer-generated input) highly accessible to youth.

In 2010, nearly $40 \%$ of US students in grades 9 to 12 exergamed at least 1 day per week [10], $25 \%$ of Canadian youth aged 15 to 18 years reported exergaming in the past week [11], and up to $20 \%$ of young adults exergamed 1 to 3 times per month or more [12]. In addition, in 2014, a representative sample of 20,122 Canadian youth in grades 6 to 10 reported exergaming for an average of $30 \mathrm{~min}$ per day [13]. The Xbox 360's Kinect unit (ie, a motion-sensing device used to capture bodily movement and promote PA) sold approximately 25 million units in 2013 [14], and Pokémon Go, released on July 6, 2016 by Nintendo, had 25 million active players daily after only 1 month of the official release [15]. Leveraging benefit from screen fervor by incorporating PA into video gaming and designing exergames [16-19] that are easily available and appealing holds promise in terms of increasing PA and reducing sedentary time [16-19]. Exergaming may be a healthier pastime than traditional video games because they elicit more energy expenditure (EE) [20-22], and they may improve physical fitness, body composition, and cognitive health [20,23-25]. The effect of exergaming on reducing sedentary behavior has also been studied, but to a much lesser extent [26]. Overall, the data suggest that exergaming may be a viable method to increase PA, change body composition, and improve mental health, and it represents an improvement in EE over sedentary behavior $[16,18,19,27]$.

There are important methodological challenges in the current literature evaluating exergaming including that many intervention studies report high attrition. Short intervention periods (ie, usually 6 or 12 weeks with no follow-up post intervention) may hinder detection of longer-term effects [24,28,29], and most studies are performed under controlled conditions that may not apply to "real-life" settings. For example, a small pilot study that provided African-American and Hispanic children with access to exergaming at home and during an after-school program indicated that fitness improved after 12 weeks [30]. However, $42 \%$ of participants lost interest in exergaming within 3 months, indicative that use related partly to the novelty of the consoles. This suggests that improving understanding of PA motivation pertaining to exergaming and sustained exergaming is needed.

\section{Objectives}

To inform the development of exergaming interventions that have the potential to be sustained, evidence is needed on the determinants of sustained exergaming in naturalistic settings. Although the study by O'Loughlin et al [12]. reported that exergamers were more likely to be girls, to play nonactive video games, to watch TV 2 hours per day or more, to report weight-related stress, and to be nonsmokers, no reports to date describe factors associated with sustained exergaming in "real-world" contexts. The objectives of this study were (1) to describe the sustainability of exergaming over 2 years in a population-based sample of adolescents and (2) to identify factors associated with sustained exergaming.

\section{Methods}

\section{Population Sample}

Data were drawn from AdoQuest I (2005-11), a 6-year longitudinal study of grade 5 students $(n=1843$ aged $10-11$ years at baseline), which investigated the natural course of the co-occurrence of health-compromising behaviors such as smoking, PA, sedentary behavior, and substance use in children [11]. A random sample of French-language elementary schools with more than 90 students in grade 5 was recruited in the greater Montreal area. To balance representation of students in high, middle, and low socioeconomic status, all schools were first stratified into groupings defined by tertile cut-offs of a school deprivation indicator based on maternal education, parental employment, and a measure of low family income that accounts for family size and area of residence [30]. An equal number of schools was then selected into each grouping, and 10 schools in the first, 10 in the second, and 9 in the third grouping $(72.5 \%(29 / 40)$ of schools invited) agreed to participate. All students in all grade 5 classes in the 29 participating schools were eligible for recruitment. Of 2946 grade 5 students, 61.13\% (1801/2946) participated at baseline (42 students joined after baseline data were collected). No data were collected from students who did not participate. Characteristics of the AdoQuest sample were comparable to those of 2 provincially representative samples of similarly aged Québec youth [31]. Parents completed mailed self-report questionnaires in 2006-07 and again in 2008-09. Participants provided assent, and their parents or guardians provided informed consent. The study received approval from the ethics and protection review boards of Concordia University and the Centre de Recherche du Centre Hospitalier de l'Université de Montréal.

Sustainability of exergaming over 2 years was investigated using data collected in the fall and winter of 2008-09 (Time 1 [T1]) when most participants were in grades 8 and 9 (aged 12-14 years) and 2 years later in the fall and winter of 2010-11 (Time 2 [T2]) when most participants were in grades 10 and 11 (aged 14-19 years). Data on exergaming and potential correlates and predictors were collected in mailed self-report questionnaires at both T1 and T2. Most questions used in AdoQuest were drawn from ongoing surveys and studies of youth including the Canadian Youth Smoking Survey [32] and the Nicotine Dependence in Teens study [33].

\section{Study Variables}

\section{Sustained Exergaming}

At T1, participants were asked: "How many hours a day do you play active video games?" Response choices included $0<1$, $\geq 1-2, \geq 2-<3, \geq 3-4$, or $\geq 5$ hours per day. Participants were categorized as exergamers if they responded $\geq 1$ hour per day. At T2, participants were asked: "Do you play active video games (eg, Wii Fit, Dance Dance, and Revolution)?” (yes, no). Participants were categorized into 1 of 2 groups based on their responses to these 2 questions. "Sustained exergamers" included 
participants who reported exergaming at $\mathrm{T} 1$ and $\mathrm{T} 2$; those who "stopped exergaming" reported exergaming at $\mathrm{T} 1$ but not $\mathrm{T} 2$. "Never exergamers" and those who began exergaming between $\mathrm{T} 1$ and $\mathrm{T} 2$ were not included in the analysis.

\section{Frequency, Timing, and Intensity of Exergaming}

At T2, questions on exergaming were included based on the International Physical Activity Questionnaire (IPAQ), a short, self-administered questionnaire used in cross-national monitoring of usual weekly PA in youth and adults. The IPAQ demonstrates reliability as well as validity against accelerometer [34]. Specifically, exergamers were asked the following questions:

1. How many days a week do you play active video games? (1-7 days)

2. How many minutes (on average) do you play each time? (open-ended)

3. Physical effort during play? (light, moderate, vigorous)

Potential correlates and predictors of sustained exergaming were selected based on factors known to be associated with PA or exergaming in adolescence [12,35] and on the availability of data in AdoQuest.

Potential predictors measured before or at $\mathrm{T} 1$ included lifestyle behaviors (ie, level of PA, ever smoked cigarettes, binge drank, marijuana use, hours of TV per day, hours of computer per day, hours of nonactive video games per day, hours of sedentary behavior per day), weight-related indicators (ie, body mass index [BMI], stress about weight, perceived weight too heavy, trying to lose weight, body-related guilt, body-related shame), depressive symptoms, and the Pediatric Daytime Sleepiness Scale (PDSS) [36].

Potential correlates measured at T2 included sociodemographic characteristics (ie, age, sex, mother university-educated, annual household income, participant currently employed), lifestyle behaviors (ie, moderate-to-vigorous PA [MVPA] per week, meeting MVPA guidelines, past-year binge drinking, past-year marijuana use, hours of TV per day, hours of computer per day, hours of nonactive video games per day), weight-related indicators (BMI, stress about weight, perceived weight too heavy, trying to lose weight, body-related guilt, body-related shame), PA motivation, and depressive symptoms. The only variable reported by parents was mother's education; all other variables were reported by participants. Multimedia Appendix
1 describes each variable in detail including response options, coding for analysis, and Cronbach alpha for scales.

\section{Data Analysis}

Descriptive statistics were used to compare participants who did and did not sustain exergaming. The analysis considered investigation of each potential correlate or predictor as an independent study that addressed a specific hypothesis so that only 3 statistical tests (ie, univariate, partially adjusted model, and fully adjusted model) were performed for each potential correlate or predictor [34]. Potential confounders were retained in the model if they were not on the causal pathway and if they were correlated with the potential correlate or predictor and outcome at $r>0.20[35,37]$. A total of 3 logistic regression models including (1) a univariate model examining the unadjusted association between the potential correlate/predictor and outcome with no covariates; (2) a partially adjusted model accounting for age and sex; and (3) a fully adjusted model including age, sex, and (other) potential confounders. Data were analyzed using SPSS version 20.0 (released 2011, SPSS Statistics for Windows; IBM Corp). All statistical tests were 2-sided, with the significance level set at 0.05 .

\section{Results}

Data on exergaming were collected in AdoQuest for the first time in grade 9 in 2008-09. Of 1801 grade 5 participants at inception in 2005-06, 68.46\% (1233/1801) completed questionnaires at T1 in grade 9, and $16.46 \%$ (203/1233) of participants reported exergaming. At T2 in grade 11, 69.01\% (1243/1801) completed questionnaires, and 23.97\% (298/1243) of participants reported exergaming. A total of $54.08 \%$ (974/1801) participants completed questionnaires at both T1 and T2. Of the 974, $62.92 \%$ (613/974) never exergamed, $17.96 \%$ (175/974) began exergaming between T1 and T2, and 8.00\% (81/974) and $10.98 \%$ (105/974) sustained and stopped exergaming, respectively. The total number of exergamers at T1 who provided data on exergaming at T2 was $19.09 \%$ (186/974), and these participants constituted the analytic sample. Table 1 compares the characteristics of the 186 participants retained in the analytic sample with those of the 59 participants who exergamed at $\mathrm{T} 1$ but were missing data on exergaming at T2. There were no statistically significant differences between the 2 groups, although $49.46 \%(92 / 186)$ of those retained reported taking action to change their weight compared with $36.02 \%(21 / 59)$ of those not retained $(P=.08)$. 
Table 1. Comparison of baseline characteristics of exergamers retained $(n=186)$ and not retained $(n=59)$ in the analytic sample, AdoQuest $2005-12$.

\begin{tabular}{|c|c|c|c|}
\hline Variable & Retained & Not retained ${ }^{\mathrm{a}}$ & $P$ value \\
\hline Age (years) at baseline, mean (SD) & $10.8(0.5)$ & $10.7(0.5)$ & .52 \\
\hline Male, n (\%) & $90(48.6)$ & $32(53.4)$ & .52 \\
\hline Mother university-educated, n (\%) & $56(30.1)$ & $15(25.5)$ & .13 \\
\hline French-speaking, n (\%) & $165(88.6)$ & $51(86.4)$ & .66 \\
\hline Self-esteem, mean (SD) & $2.0(0.7)$ & $2.0(0.7)$ & $>.99$ \\
\hline Depressive symptoms, mean (SD) & $2.0(0.7)$ & $2.0(0.6)$ & .87 \\
\hline School connectedness, mean (SD) & $2.3(1.0)$ & $2.3(0.9)$ & .97 \\
\hline Taking action to change weight, $\mathrm{n}(\%)$ & $92(49.4)$ & $21(35.8)$ & .08 \\
\hline $\mathrm{TV} \geq 3$ hours/day, n (\%) & $70(37.6)$ & $23(39.7)$ & .78 \\
\hline Perceived academic performance, $\mathrm{n}(\%)$ above average & $72(38.8)$ & $27(45.5)$ & .35 \\
\hline
\end{tabular}

${ }^{\mathrm{a}}$ Exergamers at $\mathrm{T} 1$ without exergaming data at $\mathrm{T} 2$.

The mean age of participants in the analytic sample was 16.7 (SD 0.5) years at $\mathrm{T} 2,42 \%(522 / 1243)$ were boys, $93 \%$ $(1156 / 1243)$ were white, $76 \%(945 / 1243)$ were in grade 11 , $47 \%(584 / 1243)$ were employed full or part-time, and $33 \%$ (410/1243) had university-educated mothers. The mean (SD) BMI was 23.6 (5.0) in boys and 22.2 (4.1) in girls. A total of $30 \%(373 / 1243)$ of participants' parents reported an annual household income greater or equal to Can $\$ 100,000$.

Of the 186 participants retained in the analytic sample, $43.6 \%$ (81/186) sustained exergaming (ie, reported exergaming at T1 and T2). At T2, sustained exergamers $(\mathrm{n}=81)$ exergamed on a mean (SD) of 1.9 (1.4) days per week, for 49.0 (33.8) min per bout on average. Overall, $35 \%$ (65/186) of sustained exergamers reported that they exergamed at light intensity, $42 \%(78 / 186)$ exergamed at moderate intensity, and 23\% (32/186) exergamed at vigorous intensity.

In univariate analyses of potential correlates measured at T2 (Table 2), girls were twice as likely to sustain exergaming as boys. With each unit increase in depressive symptoms, the odds of sustained exergaming increased by $40 \%$. Similarly, the odds of sustained exergaming were higher in participants with higher levels of introjected, identified, or intrinsic regulation. Finally, the odds of exergaming increased by $30 \%$ with each unit increase in body-related guilt. In partially adjusted models, girls were more likely to sustain exergaming, and for each unit increase in introjected regulation, there was a $60 \%$ increase in the odds of sustained exergaming. In fully adjusted models, the only variable retained as statistically significant was introjected regulation (odds ratio [OR] 1.8; 95\% CI 1.1-3.2). 
Table 2. Odds ratio (OR) and 95\% CI for the association between potential correlates and sustained exergaming, AdoQuest 2005-12 (N=186).

\begin{tabular}{llllll}
\hline Indicators $^{\mathrm{a}}$ & $\mathrm{n}\left(\%^{\mathrm{b}}\right)$ & $\begin{array}{l}\text { Sustained } \\
\text { exergam- } \\
\mathrm{ing}^{\mathrm{c}}, \mathrm{n}(\%)\end{array}$ & $\begin{array}{l}\mathrm{OR}_{\text {crude }} \\
(95 \% \mathrm{CI})\end{array}$ & $\begin{array}{l}\text { Model adjusted } \\
\text { for age and sex } \\
\mathrm{OR}_{\mathrm{adj}}(95 \% \mathrm{CI})\end{array}$ & $\begin{array}{l}\text { Fully adjusted } \\
\text { model OR }\end{array}$ \\
\hline
\end{tabular}

\section{Sociodemographic indicators}

Age (years) ${ }^{\text {d }}$

$\begin{array}{lllllll}14.39-16.63 & 60(32.3) & 25(41.7) & 1.3(0.8-2.1) & 1.2(0.7-2.0) & 1.2(0.7-2.0) & \text { Sex } \\ 16.64-16.98 & 59(31.7) & 21(40.7) & -\mathrm{e} & - & - & - \\ 16.99-19.86 & 59(31.7) & 28(47.5) & - & - & - & -\end{array}$

Sex

$\begin{array}{lllllll}\text { Boys } & 83(44.6) & 30(34.9) & \text { reference } & \text { reference } & \text { reference } & \text { Age, } \text { MVPA }^{\mathrm{f}}, \text { nonactive video games } \\ \text { Girls } & 103(55.4) & 51(50.5) & 2.0(1.1-3.4) & 1.9(1.0-3.4) & 1.6(0.8-3.3) & -\end{array}$

Mother university-educated

$\begin{array}{lllllll}\text { Yes } & 49(26.3) & 23(46.9) & \text { reference } & \text { reference } & \text { reference } & \text { Age, sex, income } \\ \text { No } & 108(58.1) & 45(41.7) & 0.8(0.4-1.6) & 0.8(0.4-1 .) & 0.9(0.4-2.0) & -\end{array}$

\section{Income, Can \$}

$\begin{array}{lllllll}<100 \mathrm{~K} & 84(45.2) & 33(38.9) & \text { reference } & \text { reference } & \text { reference } & \text { Age, sex, mother's education } \\ \geq 100 \mathrm{~K} & 60(32.3) & 28(46.9) & 1.4(0.7-3.0) & 1.4(0.7-3.0) & 1.5(0.7-3.2) & \text { Age, sex, mother's education }\end{array}$

\section{Employed (AdoQuest participant)}

$\begin{array}{lllllll}\text { Yes } & 78(42.0) & 34(43.6) & \text { reference } & \text { reference } & \text { reference } & \text { Age, sex } \\ \text { No } & 101(54.3) & 43(42.6) & 1.0(0.5-1.7) & 1.0(0.6-1.9) & 1.0(0.6-1.9) & \text { Age, sex }\end{array}$

\section{Lifestyle behaviors}

\section{MVPA/min per week ${ }^{d}$}

$\begin{array}{lllllll}0-105 & 59(32.8) & 21(35.6) & 1.0(1.0-1.0) & 1.0(1.0-1.0) & 1.0(1.0-1.0) & \text { Age, sex, identified regulation } \\ 106-295 & 57(30.6) & 30(52.6) & - & - & - & - \\ \geq 295 & 64(34.4) & 28(43.8) & - & - & - & -\end{array}$

\section{Meets MVPA guidelines}

$\begin{array}{lllllll}\text { Yes } & 42(22.6) & 16(38.1) & \text { reference } & \text { reference } & \text { reference } & \text { Age, sex, intrinsic, body shame } \\ \text { No } & 138(74.2) & 63(45.7) & 1.4(0.7-2.8) & 1.1(0.5-2.3) & 1.3(1.0-1.6) & -\end{array}$

Binge drank past year

$\begin{array}{lllllll}\text { Yes } & 82(44.1) & 37(45.1) & \text { reference } & \text { reference } & \text { reference } & \text { Age, sex, marijuana use, intrinsic regulation } \\ \text { No } & 94(50.5) & 39(41.5) & 0.9(0.5-1.6) & 0.9(0.5-1.6) & 1.2(0.6-2.4) & -\end{array}$

\section{Used marijuana past year}

$\begin{array}{lllllll}\text { Yes } & 141(75.8) & 71(50.0) & \text { reference } & \text { reference } & \text { reference } & \text { Age, sex, binge drinking, smoked cigarettes } \\ \text { No } & 34(18.3) & 14.2(41.8) & 1.0(0.8-1.3) & 0.7(0.3-1.6) & 0.4(0.1-1.0) & -\end{array}$

\section{Hours of TV/day ${ }^{d}$}

$\begin{array}{lllllll}\geq 0<1 & 42(22.6) & 19(45.2) & 1.0(0.8-1.4) & 1.1(0.8-1.4) & 1.1(0.8-1.5) & \text { Age, sex, depressive symptoms } \\ \geq 1<2 & 78(41.9) & 31(39.7) & - & - & - & - \\ \geq 2 & 66(35.5) & 31(47.0) & - & - & - & -\end{array}$

Hours of computer/day ${ }^{d}$

\begin{tabular}{lllllll}
$\geq 0<1$ & $38(20.4)$ & $15(39.5)$ & $1.1(0.9-1.3)$ & $1.1(0.8-1.3)$ & $1.1(0.8-1.3)^{\mathrm{g}}$ & Age, sex \\
$\geq 1<2$ & $56(30.1)$ & $22(39.3)$ & - & - & - & - \\
$\geq 2$ & $92(49.5)$ & $44(47.8)$ & - & - & - & - \\
\hline
\end{tabular}




\begin{tabular}{|c|c|c|c|c|c|c|}
\hline Indicators $^{\mathrm{a}}$ & $\mathrm{n}\left(\%^{\mathrm{b}}\right)$ & $\begin{array}{l}\text { Sustained } \\
\text { exergam- } \\
\text { ing }^{\mathrm{c}}, \mathrm{n}(\%)\end{array}$ & $\begin{array}{l}\text { OR }_{\text {crude }} \\
(95 \% \mathrm{CI})\end{array}$ & $\begin{array}{l}\text { Model adjusted } \\
\text { for age and sex } \\
\mathrm{OR}_{\mathrm{adj}}(95 \% \mathrm{CI})\end{array}$ & $\begin{array}{l}\text { Fully adjusted } \\
\text { model OR } \mathrm{OR}_{\mathrm{adj}} \\
(95 \% \mathrm{CI})\end{array}$ & Covariate(s) included in fully adjusted model \\
\hline
\end{tabular}

Hours of nonactive video games/day ${ }^{d}$

$\begin{array}{lllllll}0 & 82(44.1) & 32(39.0) & 1.0(0.8-1.3) & 1.0(0.9-1.4) & 1.0(0.9-1.4)^{\mathrm{g}} & \text { Age, sex } \\ <1 & 50(26.7) & 28(56.0) & - & - & - & - \\ \geq 1 & 53(28.5) & 21(39.6) & - & - & -\end{array}$

\section{Weight-related indicators}

\section{BMI $^{\text {d,h }}$}

$\begin{array}{lllll}16.01-20.1 & 49(26.3) & 23(46.9) & 1.0(0.9-1.0) & 0.9(0.9-1.1) \\ 20.2-23.54 & 51(27.4) & 20(39.2) & - & - \\ 23.55-40.70 & 49(26.3) & 18(36.7) & - & -\end{array}$

Stressed about weight

Yes

No

$129(69.4) \quad 52(40.3)$

$0.7(0.4-1.3) \quad 0.8(0.3-2.1)$

$0.9(0.3-2.0)$

Perceived weight status: overweight

$\begin{array}{llllll}\text { Yes } & 49(26.3) & 23(46.9) & \text { reference } & \text { reference } & \text { reference } \\ \text { No } & 127(68.3) & 53(41.7) & 0.8(0.2-1.6) & 0.8(0.2-1.6) & 0.3(0.1-1.3)\end{array}$

Trying to lose weight

Yes $\quad 114(61.3) \quad 57(50.0) \quad$ reference reference reference

No

$62(33.3) \quad 25(39.5)$

$0.7(0.4-1.2) \quad 0.8(0.4-1.4)$

$0.8(0.3-1.2)$

Body-related guilt ${ }^{\mathrm{d}}$

$0 \leq 1.0$

$67(36.0) \quad 20(29.9)$

$1.3(1.0-1.7) \quad 1.2(0.9-1.7)$

$0.9(0.4-2.0)$

$\begin{array}{lll}>1.0 \leq 2.0 & 52(28.0) & 26(50.0) \\ >2.0-5.0 & 58(31.2) & 29(50.0)\end{array}$

Body-related shame ${ }^{\mathrm{d}}$

$0 \leq 1.0$

$61(32.8) \quad 16(26.2)$

$1.2(0.9-1.7) \quad 1.2(0.9-1.6)$

$1.0(0.4-2.3)$

$\begin{array}{lll}1.0 \leq 2.0 & 59(31.7) & 31(52.5) \\ >2.0-4.8 & 57(30.6) & 28(49.1)\end{array}$

\section{Physical activity motivation}

Amotivation $^{d}$

$0 \leq 1$ $130(69.9) \quad 57(43.8)$

$1.3(0.7-2.4) \quad 1.3(0.7-2.6)$

$2.0(0.9-5.0)$
Age, sex, stress about weight, BMI, depressive symptoms, weight perception, extrinsic regulation, introjected regulation, body guilt, trying to lose weight

Age, sex, income, extrinsic motivation, body shame, body guilt

Age, sex, depressive symptoms, weight perception, introjected regulation, external regulation, body shame, body guilt, trying to lose weight

Age, sex, BMI, introjected regulation, external regulation, body shame, body guilt, stress about weight, trying to lose weight

Age, sex, BMI, weight perception, introjected regulation, external regulation, identified regulation, stress about weight, weight perception, body shame, body guilt

Age, sex, BMI, depressive symptoms, weight perception, extrinsic regulation, introjected regulation, identified regulation, body shame, stress about weight, trying to lose weight

-

-

$-$
Age, sex, identified regulation, intrinsic regulation, body shame 


\begin{tabular}{llllll}
\hline Indicators $^{\mathrm{a}}$ & $\mathrm{n}\left(\%^{\mathrm{b}}\right)$ & $\begin{array}{l}\text { Sustained } \\
\text { exergam- } \\
\mathrm{ing}^{\mathrm{c}}, \mathrm{n}(\%)\end{array}$ & $\begin{array}{l}\mathrm{OR}_{\text {crude }} \\
(95 \% \mathrm{CI})\end{array}$ & $\begin{array}{l}\text { Model adjusted } \\
\text { for age and sex } \\
\mathrm{OR}_{\text {adj }}(95 \% \mathrm{CI})\end{array}$ & $\begin{array}{l}\text { Fully adjusted } \\
\text { model OR }\end{array}$ \\
& & $95 \% \mathrm{CI})$ & Covariate(s) included in fully adjusted model \\
\hline
\end{tabular}

External $^{\text {d }}$

$0 \leq 1$

$113(60.8) \quad 46(40.7)$

$1.0(0.5-2.1) \quad 1.1(0.5-2.2)$

$0.9(0.3-2.8)$

$>1 \leq 1.67$

$$
20 \text { (10.8) }
$$

11 (55)

1.68-3.25

$44(23.7)$

$18(40.9)$

Introjected $^{d}$

0

$67(36.0) \quad 21(31.3)$

$1.7(1.2-2.4) \quad 1.6(1.1-2.3)$

$1.8(1.1-3.2)$

$1-1.67$
$1.68-5$

46 (24.7)

$64(34.4)$

20 (43.5)

$24(53.1)$

Identified $^{d}$

0-2.25

$54(29.0)$

$29(37.0)$

$1.4(1.0-1.8) \quad 1.3(1.0-1.8)$

$1.3(0.8-2.0)$

2.26-3.25

$63(33.9)$

22 (34.9)

3.26-5

$60(32.3)$

$33(55.0)$

Intrinsic $^{d}$

0-1.0

$56(30.1)$

$19(33.9)$

$1.3(1.0-1.6) \quad 1.3(1.0-1.7)$

$1.3(0.9-2.0)$

$\begin{array}{lll}1-1.67 & 55(29.6) & 23(41.8) \\ \geq 1.68 & 66(35.5) & 33(50.0)\end{array}$

\section{Depressive symptoms $^{d}$}

$0-1.67$

$59(31.7)$

$22(37.3)$

$1.4(1.0-2.0) \quad 1.3(0.9-1.9)$

$1.4(0.8-2.2)$

Age, sex, introjected regulation, external regulation, body shame, body guilt, stress about weight, TV use
Age, sex, MVPA, amotivation, identified, binge drinking, introjected regulation, body shame

Age, sex. BMI, depressive symptoms, stress about weight, weight perception, trying to lose weight, introjected regulation, body shame, body guilt

Age, sex, depressive symptoms, weight stress, weight perception, external regulation, identified regulation, intrinsic regulation, trying to lose weight, body shame, body guilt

Age, sex, amotivation, introjected regulation, intrinsic motivation, regulation, body guilt, trying to lose

-

$\begin{array}{lllllll}1.68-2.50 & 66(35.5) & 25(37.9) & - & - & - & - \\ 2.51-4.5 & 54(29.0) & 30(55.6) & - & - & - & -\end{array}$

${ }^{\mathrm{a}}$ Potential correlate measured in grade 11.

${ }^{b}$ Percentages may not total $100 \%$ due to missing data.

${ }^{\mathrm{c}}$ Continuous variables were grouped according to tertile cut-offs for descriptive purposes.

${ }^{\mathrm{d}}$ Potential correlate included in the model as a continuous variable. Odds ratio indicates the increase in the probability of the outcome per 1-unit change in the correlate.

${ }^{\mathrm{e}}$ Not applicable.

fMVPA: moderate-to-vigorous physical activity.

${ }^{\mathrm{g}}$ Partially and fully adjusted models were identical.

${ }^{\mathrm{h}}$ BMI: body mass index.

In univariate analyses of potential predictors (Table 3), more time spent playing nonactive video games daily was protective against sustained exergaming sustainers. Trying to lose weight was associated with an $80 \%$ increase in the odds of sustained exergaming. In partially adjusted models controlling for sex and age and in fully-adjusted models, none of the potential predictors were statistically significantly associated with sustained exergaming. 
Table 3. Odds ratio (OR) and 95\% CI for the association between potential predictors and sustained exergaming, AdoQuest 2005-12 (N=186).

\begin{tabular}{llllll}
\hline Predictors $^{\mathrm{a}}$ & $\mathrm{n}\left(\%^{\mathrm{b}}\right)$ & $\begin{array}{l}\text { Sustained } \\
\text { exergam- } \\
\mathrm{ing}^{\mathrm{c}}, \mathrm{n}(\%)\end{array}$ & $\begin{array}{l}\mathrm{OR}_{\text {crude }} \\
(95 \% \mathrm{CI})\end{array}$ & $\begin{array}{l}\text { Model adjusted } \\
\text { for age and sex, } \\
\mathrm{OR}_{\mathrm{adj}}(95 \% \mathrm{CI})\end{array}$ & $\begin{array}{l}\text { Fully adjusted } \\
\text { model, OR }\end{array}$ \\
\hline
\end{tabular}

\section{Lifestyle behaviors}

Physical activity level $^{\mathrm{d}}$

$\begin{array}{lllllll}1 & 37(20.0) & 13(35.1) & 1.0(0.8-1.3) & 1.1(0.8-1.3) & 1.1(0.8-1.3)^{\mathrm{e}} & \text { Age, sex } \\ 2-3 & 107(57.5) & 50(46.7) & -\mathrm{f} & - & - & - \\ 4-5 & 40(21.3) & 16(40.0) & - & - & - & -\end{array}$

\section{Ever smoked cigarettes}

$\begin{array}{lllllll}\text { Yes } & 57(30.7) & 29(50.9) & \text { reference } & \text { reference } & \text { reference } & \begin{array}{l}\text { Age, sex, cannabis, binge drinking, depres- } \\ \text { sive symptoms, PDSS }\end{array} \\ \text { No } & 127(68.3) & 51(40.2) & 0.7(0.4-1.2) & 0.7(0.4-1.3) & 0.5(0.3-1.2) & -\end{array}$

Binge drank

$\begin{array}{lllllll}\text { Yes } & 43(26.3) & 16(37.2) & \text { reference } & \text { reference } & \text { reference } & \begin{array}{l}\text { Age, sex, cigarette, depressive symptoms, } \\ \text { cannabis, PDSS }\end{array} \\ \text { No } & 143(76.9) & 65(45.5) & 1.4(0.7-2.8) & 1.3(0.6-2.6) & 1.5(0.6-3.8) & -\end{array}$

Marijuana use

$\begin{array}{lllllll}\text { Yes } & 21(11.3) & 9(42.9) & \text { reference } & \text { reference } & \text { reference } & \begin{array}{l}\text { Age, sex, cig try, binge drinking, depressive } \\ \text { symptoms }\end{array} \\ \text { No } & 164(88.2) & 72(43.9) & 1.0(0.4-2.6) & 0.9(0.4-2.4) & 1.0(0.3-3.5) & \text { - }\end{array}$

Hours of TV/day

$\begin{array}{lllllll}\leq 1 & 20(10.8) & 9(45.0) & 1.1(0.6-2.2) & 0.8(0.6-1.1) & 0.9(0.6-1.4) & \text { Age, sex, computer, sedentary behavior } \\ \geq 1 \leq 3 & 93(50.0) & 41(44.1) & - & - & - & - \\ >3 & 72(38.8) & 30(41.7) & - & - & - & -\end{array}$

Hours of computer/day ${ }^{d}$

$\begin{array}{lllllll}\leq 1 & 31(16.7) & 11(35.5) & 1.3(1.0-1.7) & 1.4(1.0-2.0) & 1.3(0.9-2.1) & \begin{array}{l}\text { Age, sex, depressive symptoms, video games, } \\ \text { TV, sedentary behavior }\end{array} \\ \geq 1 \leq 3 & 75(40.3) & 29(38.7) & - & - & - & - \\ >3 & 79(42.5) & 41(51.9) & - & - & - & -\end{array}$

Hours of nonactive video games/day ${ }^{d}$

$\begin{array}{lllllll}\leq 1 & 107(57.5) & 54(50.5) & 0.6(0.5-1.0) & 0.8(0.5-1.3) & 0.8(0.4-1.3) & \text { Age, sex, computer, sedentary behavior } \\ >1 & 73(39.2) & 26(35.6) & - & - & - & -\end{array}$

\section{Hours of sedentary behavior/day ${ }^{\mathrm{d}}$}

$\begin{array}{lllllll}0-9.5 & 60(32.3) & 23(38.3) & 1.1(1.0-1.2) & 1.0(1.0-1.1) & 1.0(0.9-1.2) & \text { Age, sex, TV, computer, video games } \\ 9.6-11.5 & 52(27.4) & 24(46.2) & - & - & - & - \\ >11.5 & 61(37.8) & 30(49.2) & - & - & - & -\end{array}$

\section{Weight-related indicators}

\section{BMI $^{\text {d,h }}$}

\begin{tabular}{|c|c|c|c|c|c|c|}
\hline $13.7-19.2$ & $35(18.8)$ & 17 (48.6) & $1.0(0.9-1.1)$ & $1.1(1.0-1.2)$ & $1.1(1.0-1.2)$ & $\begin{array}{l}\text { Age, sex, trying to lose weight, video games, } \\
\text { perceived weight, stress about weight, } \\
\text { sedentary behavior }\end{array}$ \\
\hline $19.3-22.66$ & 35 (18.8) & $14(40.0)$ & - & - & - & - \\
\hline $22.67+$ & $36(19.4)$ & $11(30.6)$ & - & - & - & - \\
\hline
\end{tabular}




\begin{tabular}{|c|c|c|c|c|c|c|}
\hline Predictors $^{\mathrm{a}}$ & $\mathrm{n}\left(\%^{\mathrm{b}}\right)$ & $\begin{array}{l}\text { Sustained } \\
\text { exergam- } \\
\text { ing }^{\mathrm{c}}, \mathrm{n}(\%)\end{array}$ & $\begin{array}{l}\text { OR }_{\text {crude }} \\
(95 \% \mathrm{CI})\end{array}$ & $\begin{array}{l}\text { Model adjusted } \\
\text { for age and sex, } \\
\mathrm{OR}_{\text {adj }}(95 \% \mathrm{CI})\end{array}$ & $\begin{array}{l}\text { Fully adjusted } \\
\text { model, OR adj } \\
(95 \% \mathrm{CI})\end{array}$ & Covariates included in fully adjusted model \\
\hline Yes & $80(43.0)$ & $40(50.0)$ & reference & reference & reference & $\begin{array}{l}\text { Age, sex, depressive symptoms, trying to lose } \\
\text { weight, perceived weight, PDSS, BMI }\end{array}$ \\
\hline No & $105(56.5)$ & $41(39.0)$ & $0.6(0.4-1.2)$ & $0.8(0.4-1.5)$ & $0.6(0.2-1.8)$ & - \\
\hline
\end{tabular}

Perceived weight too heavy

$\begin{array}{lllllll}\text { Yes } & 55(29.6) & 16(29.5) & \text { reference } & \text { reference } & \text { reference } & \begin{array}{l}\text { Age, sex, BMI, stress about weight, trying to } \\ \text { lose weight }\end{array} \\ \text { No } & 128(68.8) & 39(30.8) & 1.1(0.6-2.1) & 1.1(0.6-2.2) & 3.0(0.8-10.8) & \text { - }\end{array}$

Trying to lose weight

$\begin{array}{lllllll}\text { No } & 113(60.8) & 42(37.2) & \text { reference } & \text { reference } & \text { reference } & \begin{array}{l}\text { Age, sex, stress about weight, depressive } \\ \text { symptoms, perceived weight }\end{array} \\ \text { Yes } & 70(37.6) & 36(51.4) & 1.8(1.0-3.3) & 1.3(0.7-2.6) & 1.6(0.7-3.5) & \text { - }\end{array}$

Depressive symptoms ${ }^{d}$

$\begin{array}{lllllll}0-1.5 & 57(30.6) & 27(47.4) & 1.0(0.7-1.4) & 0.8(0.5-1.2) & 0.8(0.5-1.3) & \begin{array}{l}\text { Age, sex, stress weight, binge drinking, trying } \\ \text { to lose weight, PDSS, cigarettes, computer, } \\ \text { sedentary behavior }\end{array} \\ \begin{array}{llllll}1.6-1.17 \\ 1.8-5.0\end{array} & 65(35.0) & 24(36.9) & - & - & - & - \\ \text { SS }^{\mathbf{d}} & 62(33.3) & 30(48.4) & - & - & - & \text { Age, sex, stress about weight, binge drinking, } \\ 0-7 & 62(33.3) & 24(38.7) & 1.0(1.0-1.1) & 1.0(1.0-1.1) & 1.0(1.0-1.1) & \text { depressive symptoms } \\ 8-13 & 60(32.3) & 23(38.3) & - & - & - & - \\ \geq 14 & 62(33.3) & 34(54.8) & - & - & - & -\end{array}$

\footnotetext{
${ }^{\mathrm{a}}$ Potential predictor measured in grade 9 .

${ }^{\mathrm{b}}$ Percentages may not total $100 \%$ due to missing data. change in the predictor.

${ }^{\mathrm{e}}$ Partially and fully adjusted models were identical.

${ }^{\mathrm{f}}$ Not applicable.

${ }^{g}$ PDSS: Paediatric daytime sleepiness scale.

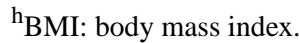

${ }^{\mathrm{c}}$ Continuous variables were grouped according to tertile cut-offs for descriptive purposes.

${ }^{\mathrm{d}}$ Potential predictor included in the models as a continuous variable. Odds ratio (OR) indicates the increase in the probability of the outcome per 1 unit

\section{Discussion}

\section{Principal Findings}

The purpose of this study was to describe sustained exergaming in a population-based sample of adolescents and to identify factors associated with sustained exergaming. To our knowledge, this is the first investigation of sustained exergaming in a population-based sample. Results indicated that $44 \%$ of exergamers sustained exergaming for at least 2 years. Female sex and having higher introjected PA behavior regulation were associated with sustained exergaming.

In a cross-sectional study of 200 children who owned consoles, Simons et al [38] found that $11 \%$ never exergamed and only $32 \%$ exergamed regularly (ie, $>1$ hour per week). Because of concerns that the novelty of exergaming dissipates over time, there have been numerous calls to investigate the sustainability of exergaming [17,24,28,39-42]. The $44 \%$ (81/186) of sustained exergamers in our study compares with the $41 \%$ of girls (but not the $69 \%$ of boys) who remained involved in team sports for over 5 years, as observed by Belanger et al [41]. Specifically, of 1276 adolescents age 12-13 years who completed a 7-day PA recall every 3 months for 5 years, the authors reported that between $14 \%$ and $53 \%$ re-engaged in a specific PA after discontinuation. Although not investigated in the Belanger study, exergaming could be an intermittent activity in adolescents linked to the release of new games and consoles. Future studies will need more frequent follow-up to assess the stop-start aspect of exergaming over time.

Three-quarters (77\% [143/186]) of participants exergamed at light or moderate intensity. Exergaming at these intensities may be more enjoyable, practical, and achievable among young persons. Thus, the flexibility of exergaming in level of intensity may contribute to sustainability. 
Previous studies have reported that females are more likely to exergame than males [12], and in this analysis, girls were more likely to sustain exergaming. The reasons for sex differences in sustained exergaming may mirror the reasons for sex difference in exergaming. Girls, especially those with body image challenges, may be more comfortable being active at home away from the scrutiny of others, while still enjoying the social interaction provided by exergaming. Boys may be attracted by the novelty of exergaming but return to nonactive video games as the novelty wears off. Although sex differences in exergaming should be further investigated, our results suggest that exergaming may be a viable option to help girls in particular remain physically active.

A specific focus of this work was to assess whether PA motivation is associated with sustained exergaming. Self-determination theory (SDT) has been used as a framework to predict intentions to engage in and sustain traditional PA, and investigators have reported that those with higher intrinsic regulation (ie, motivation) report increased intentions to engage in PA than those with external PA regulation (eg, [43-46]. Introjected regulation (ie, a type of PA motivation indicative of internalization of PA as a behavior) was a correlate of sustained exergaming. Introjected regulation, as described in the SDT [47-49], is motivation from an internal pressure that usually drives short-term behavior change, but does not foster sustainable behavior change $[48,50]$. It is generally negatively associated with or unrelated to PA levels, although there appears to be sex differences such that girls who report higher introjected regulation also report more PA [49]. The link between behavior regulation and exergaming has been studied in clinical settings and in specific populations such as overweight youth [51,52], but few studies investigate this association in population-based samples of youth using the SDT [53], and it is not fully understood if exercise behavior regulation differs between those who do and do not sustain exergaming. Sustained exergamers may not be highly motivated intrinsically toward traditional PA and are drawn toward exergaming as a PA alternative. They may be interested in developing skills for fitness and weight change, which is fostered through fitness exergames (ie, body weight, body alignment, placement, feeling, and speed captured by motion and sensor-captures and displayed in real time on the screen) [52], or aspects of the advertising or marketing of fitness exergames may play on or increase guilt, particularly among female users. Future research should focus on better understanding the differences between motivation for PA and for exergaming, which in turn lead to exergaming interventions that have a greater impact on PA and sedentary behavior.

No predictors of sustained exergaming were identified in this study. Rather than being a planned behavior, exergaming may be triggered by events in the immediate present such as purchasing a new console or friends coming over, and therefore, has few predictors. It is also possible that the sample size was too small to detect factors associated with sustained exergaming because exergaming is a relatively new area of research, not all relevant predictors may not have been investigated.

Limitations of this study include the small sample size, that self-report data are subject to misclassification, that loss to follow-up may have resulted in selection bias, and that restriction of the sample to francophones may have limited external generalizability. As data were not collected more frequently, it was not possible to confirm whether participants exergamed between data collection cycles.

\section{Conclusions}

Exergaming may represent a novel approach to help adolescents remain physically active during a period of life which is notable for sharp declines in PA. Exergaming may be more sustainable if games include components that foster intrinsic PA motivation such as providing more choices in the games offered (ie, with whom the game can be played, whether the setting is collaborative or competitive, how intensely the game is played) or having a coach and/or other social support while learning a new game. Finally, clinicians and practitioners can counsel parents to encourage their children to choose exergaming over more traditional sedentary video games or to exergame as a family.

\section{Acknowledgments}

This project was funded by the Canadian Tobacco Control Research Initiative and the Institut national de santé publique du Québec (INSPQ) through a financial contribution from the Québec Ministry of Health and Social Services to the INSPQ. Views expressed in this document do not necessarily reflect those of the Québec Ministry of Health and Social Services. This work is part of the first author's (EKO) PhD dissertation and coauthors are committee members.

\section{Conflicts of Interest}

None declared.

\section{Multimedia Appendix 1}

Variables tested in models including response options, coding for analysis, and Cronbach alpha for scales.

[PDF File (Adobe PDF File), 90KB-Multimedia Appendix 1]

\section{References}

1. Colley R, Garriguet D, Janssen I, Craig CL, Clarke J, Tremblay MS. Physical activity of Canadian children and youth: accelerometer results from the 2007 to 2009 Canadian Health Measures Survey. Health Rep 2011 Mar;22(1):15-23 [FREE Full text] [Medline: 21510586] 
2. Rideout V, Foehr UG, Roberts DF. Education Resources Information Center.: ERIC Clearinghouse; 2010. Generation M: Media in the Lives of 8-to 18-Year-Olds URL: https://eric.ed.gov/?id=ED527859 [accessed 2019-04-22] [WebCite Cache ID 77pb0ULzj]

3. Janssen I, LeBlanc AG. Systematic review of the health benefits of physical activity and fitness in school-aged children and youth. Int J Behav Nutr Phys Act 2010;7(1):40-16. [doi: 10.1186/1479-5868-7-40]

4. Warburton D, Nicol CW, Bredin SS. Health benefits of physical activity: the evidence. Can Med Assoc J 2006 Mar 14;174(6):801-809 [FREE Full text] [doi: 10.1503/cmaj.051351] [Medline: 16534088]

5. Tremblay M, LeBlanc AG, Kho ME, Saunders TJ, Larouche R, Colley RC, et al. Systematic review of sedentary behaviour and health indicators in school-aged children and youth. Int J Behav Nutr Phys Act 2011 Sep 21;8(1):98 [FREE Full text] [doi: 10.1186/1479-5868-8-98] [Medline: 21936895]

6. Patnode CD, Lytle LA, Erickson DJ, Sirard JR, Barr-Anderson DJ, Story M. Physical activity and sedentary activity patterns among children and adolescents: a latent class analysis approach. J Phys Act Health 2011 May;8(4):457-467 [FREE Full text] [Medline: 21597117]

7. Must A, Tybor DJ. Physical activity and sedentary behavior: a review of longitudinal studies of weight and adiposity in youth. Int J Obes (Lond) 2005 Sep;29(Suppl 2):S84-S96. [Medline: 16385758]

8. Ortega F, Konstabel K, Pasquali E, Ruiz JR, Hurtig-Wennlöf A, Mäestu J, et al. Objectively measured physical activity and sedentary time during childhood, adolescence and young adulthood: a cohort study. PLoS One 2013;8(4):e60871 [FREE Full text] [doi: 10.1371/journal.pone.0060871] [Medline: 23637772]

9. Verloigne M, Van Lippevelde W, Maes L, Yıldırım M, Chinapaw M, Manios Y, et al. Levels of physical activity and sedentary time among 10- to 12-year-old boys and girls across 5 European countries using accelerometers: an observational study within the ENERGY-project. Int J Behav Nutr Phys Act 2012 Mar 31;9:34 [FREE Full text] [doi:

10.1186/1479-5868-9-34] [Medline: 22462550]

10. Madden M, Lenhart A, Duggan M, Cortesi S, Gasser U. Pew Research Center. 2013. Teens and technology 2013 URL: https://www.pewinternet.org/wp-content/uploads/sites/9/media/Files/Reports/2013/PIP TeensandTechnology2013.pdf [accessed 2019-04-22] [WebCite Cache ID 77plVVewg]

11. Fulton J. Circulation. 2010. Abstract P260: Active Video Game Participation in US Youth: Findings from the National Youth Physical Activity and Nutrition Survey, 2010 URL: https://www.ahajournals.org/doi/abs/10.1161/circ.125.suppl 10. ap260 [accessed 2019-04-26] [WebCite Cache ID 77vsIHzHZ]

12. O'Loughlin E, Dugas EN, Sabiston CM, O'Loughlin JL. Prevalence and correlates of exergaming in youth. Pediatrics 2012 Nov;130(5):806-814. [doi: 10.1542/peds.2012-0391] [Medline: 23027171]

13. Kakinami L, O'Loughlin EK, Dugas EN, Sabiston CM, Paradis G, O'Loughlin J. The association between exergaming and physical activity in young adults. J Phys Act Health 2015 Jun;12(6):789-793. [doi: 10.1123/jpah.2013-0447] [Medline: 25133470]

14. Janssen I. Estimating whether replacing time in active outdoor play and sedentary video games with active video games influences youth's mental health. J Adolesc Health 2016 Dec;59(5):517-522. [doi: 10.1016/j.jadohealth.2016.07.007] [Medline: 27567064]

15. Makuck E. Gamespot. 2013. Kinect Sales Reach 24 Million URL: https://www.gamespot.com/articles/ kinectsales-reach-24-million/1100-6403766/ [accessed 2019-04-23] [WebCite Cache ID 77qdOcRcL]

16. Best JR. Exergaming in youth: effects on physical and cognitive health. Z Psychol 2013 Apr 01;221(2):72-78 [FREE Full text] [doi: 10.1027/2151-2604/a000137] [Medline: 25097828]

17. Lamboglia CM, da Silva VT, de Vasconcelos Filho JE, Pinheiro MH, Munguba MC, Silva Júnior FV, et al. Exergaming as a strategic tool in the fight against childhood obesity: a systematic review. J Obes 2013;2013:438364 [FREE Full text] [doi: 10.1155/2013/438364] [Medline: 24319594]

18. Sween J, Wallington SF, Sheppard V, Taylor T, Llanos AA, Adams-Campbell LL. The role of exergaming in improving physical activity: a review. J Phys Act Health 2014 May;11(4):864-870 [FREE Full text] [doi: 10.1123/jpah.2011-0425] [Medline: 25078529]

19. Zeng N, Gao Z. Exergaming and obesity in youth: current perspectives. Int J Gen Med 2016;9:275-284 [FREE Full text] [doi: 10.2147/IJGM.S99025] [Medline: 27536158]

20. Graf D, Pratt LV, Hester CN, Short KR. Playing active video games increases energy expenditure in children. Pediatrics 2009 Aug;124(2):534-540. [doi: 10.1542/peds.2008-2851] [Medline: 19596737]

21. Lanningham-Foster L, Jensen TB, Foster RC, Redmond AB, Walker BA, Heinz D, et al. Energy expenditure of sedentary screen time compared with active screen time for children. Pediatrics 2006 Dec;118(6):e1831-e1835. [doi: 10.1542/peds.2006-1087] [Medline: 17142504]

22. Peng W, Lin JH, Crouse J. Is playing exergames really exercising? A meta-analysis of energy expenditure in active video games. Cyberpsychol Behav Soc Netw 2011 Nov;14(11):681-688. [doi: 10.1089/cyber.2010.0578] [Medline: 21668370]

23. Chen J, Wilkosz ME. Efficacy of technology-based interventions for obesity prevention in adolescents: a systematic review. Adolesc Health Med Ther 2014;5:159-170 [FREE Full text] [doi: 10.2147/AHMT.S39969] [Medline: 25177158] 
24. Guy S, Ratzki-Leewing A, Gwadry-Sridhar F. Moving beyond the stigma: systematic review of video games and their potential to combat obesity. Int J Hypertens 2011;2011:179124 [FREE Full text] [doi: 10.4061/2011/179124] [Medline: 21629863]

25. Lu A, Kharrazi H, Gharghabi F, Thompson D. A systematic review of health videogames on childhood obesity prevention and intervention. Games Health J 2013 Jun;2(3):131-141 [FREE Full text] [doi: 10.1089/g4h.2013.0025] [Medline: 24353906]

26. Gao Y, Gerling K, Mandryk R, Stanley K. Decreasing sedentary behaviours in pre-adolescents using casual exergames at school. 2014 Oct 19 Presented at: Proceedings of the first ACM SIGCHI annual symposium on Computer-human interaction in play; October 19-21, 2014; Toronto, Ontario, Canada. [doi: 10.1145/2658537.2658693]

27. Li J, Theng YL, Foo S. Effect of exergames on depression: a systematic review and meta-analysis. Cyberpsychol Behav Soc Netw 2016 Jan;19(1):34-42. [doi: 10.1089/cyber.2015.0366] [Medline: 26716638]

28. Gao Z, Chen S. Are field-based exergames useful in preventing childhood obesity? A systematic review. Obes Rev 2014 Aug;15(8):676-691. [doi: 10.1111/obr.12164] [Medline: 24602072]

29. LeBlanc A, Chaput JP, McFarlane A, Colley RC, Thivel D, Biddle SJ, et al. Active video games and health indicators in children and youth: a systematic review. PLoS One 2013;8(6):e65351 [FREE Full text] [doi: 10.1371/journal.pone.0065351] [Medline: 23799008]

30. Bethea TC, Berry D, Maloney AE, Sikich L. Pilot study of an active screen time game correlates with improved physical fitness in minority elementary school youth. Games Health J 2012 Feb;1(1):29-36 [FREE Full text] [doi: 10.1089/g4h.2011.0005] [Medline: 26196430]

31. O?Loughlin E. K.., Prevalence and correlates of exergaming in youth. Pediatrics 2012;130(5):806-814.

32. [Ministry of Education]. [National Institute of Public Health of Quebec]. 2003. [School Population Map and Deprivation Indices] URL: http://www.education.gouv.qc.ca/fileadmin/site_web/documents/PSG/statistiques_info_decisionnelle/ bulletin 26.pdf [accessed 2019-04-23] [WebCite Cache ID 77qsMkx7o]

33. Sylvestre M, Wellman RJ, O'Loughlin EK, Dugas EN, O'Loughlin J. Gender differences in risk factors for cigarette smoking initiation in childhood. Addict Behav 2017 Dec;72:144-150. [doi: 10.1016/j.addbeh.2017.04.004] [Medline: 28399489]

34. Hagströmer M, Oja P, Sjöström M. The International Physical Activity Questionnaire (IPAQ): a study of concurrent and construct validity. Public Health Nutr 2006 Sep;9(6):755-762. [Medline: 16925881]

35. Sallis J, Prochaska JJ, Taylor WC. A review of correlates of physical activity of children and adolescents. Med Sci Sports Exerc 2000 May;32(5):963-975. [Medline: 10795788]

36. Perez-Chada D, Perez-Lloret S, Videla AJ, Cardinali D, Bergna MA, Fernández-Acquier MA, et al. Sleep disordered breathing and daytime sleepiness are associated with poor academic performance in teenagers. A study using the Pediatric Daytime Sleepiness Scale (PDSS). Sleep 2007 Dec;30(12):1698-1703 [FREE Full text] [doi: 10.1093/sleep/30.12.1698] [Medline: 18246979 ]

37. Bender R, Lange S. Adjusting for multiple testing--when and how? J Clin Epidemiol 2001 Apr;54(4):343-349. [Medline: 11297884]

38. Simons M, Bernaards C, Slinger J. Active gaming in Dutch adolescents: a descriptive study. Int J Behav Nutr Phys Act 2012 Oct 02;9:118 [FREE Full text] [doi: 10.1186/1479-5868-9-118] [Medline: 23031076]

39. Barnett A, Cerin E, Baranowski T. Active video games for youth: a systematic review. J Phys Act Health 2011 Jul;8(5):724-737. [Medline: 21734319]

40. Biddiss E, Irwin J. Active video games to promote physical activity in children and youth: a systematic review. Arch Pediatr Adolesc Med 2010 Jul;164(7):664-672. [doi: 10.1001/archpediatrics.2010.104] [Medline: 20603468]

41. Carmo J, Palmeira A. Can active video games be part of the solution to promote physical activity in youth? A systematic review. Archives of Exercise in Health and Disease 2013;4(1):216-226 [FREE Full text] [doi: 10.4018/ijgcms.2014100105]

42. Kari T. Can exergaming promote physical fitness and physical activity? A systematic review of systematic reviews. Int $\mathbf{J}$ Gaming Comput Mediat Simul 2014;6(4):59-77. [doi: 10.4018/ijgcms.2014100105]

43. Edmunds J, Ntoumanis N, Duda JL. Examining exercise dependence symptomatology from a self-determination perspective. J Health Psychol 2006 Nov;11(6):887-903. [doi: 10.1177/1359105306069091] [Medline: 17035261]

44. Edmunds J, Ntoumanis N, Duda J. Testing a self-determination theory-based teaching style intervention in the exercise domain. Eur J Soc Psychol 2008 Mar;38(2):375-388. [doi: 10.1002/ejsp.463]

45. Edmunds J, Ntoumanis N, Duda J. Helping your clients and patients take ownership over their exercise: Fostering exercise adoption, adherence, and associated well-being. ACSMs Health Fit J 2009;13(3):20-25 [FREE Full text]

46. Thøgersen-Ntoumani C, Ntoumanis N. The role of self-determined motivation in the understanding of exercise-related behaviours, cognitions and physical self-evaluations. J Sports Sci 2006 Apr;24(4):393-404. [doi: 10.1080/02640410500131670] [Medline: 16492603]

47. Brötz D. Exercise, physical activity, and Self-Determination Theory: a systematic review. physioscience 2013 Feb 26;9(01):37 [FREE Full text] [doi: 10.1055/s-0032-1330638]

48. Deci E. Overview of self-determination theory: an organismic dialectical perspective. In: Ryan R, editor. Handbook of Self-Determination Research. Rochester, NY, US: University of Rochester Press; 2002:A-33. 
49. Teixeira PJ, Carraça EV, Markland D, Silva MN, Ryan RM. Exercise, physical activity, and self-determination theory: a systematic review. Int J Behav Nutr Phys Act 2012 Jun 22;9:78 [FREE Full text] [doi: 10.1186/1479-5868-9-78] [Medline: 22726453]

50. Deci E. Self-determination theory. In: Ryan R, editor. Handbook of Theories of Social Psychology. Thousand Oaks, California: Sage publications; 2011:416-433.

51. Fitzgerald D, Trakarnratanakul N, Smyth B, Caulfield B. Effects of a wobble board-based therapeutic exergaming system for balance training on dynamic postural stability and intrinsic motivation levels. J Orthop Sports Phys Ther 2010 Jan;40(1):11-19. [doi: 10.2519/jospt.2010.3121] [Medline: 20044704]

52. Osorio G, Moffat DC, Sykes J. Exergaming, exercise, and gaming: sharing motivations. Games Health J 2012 Jun;1(3):205-210. [doi: 10.1089/g4h.2011.0025] [Medline: 26193438]

53. Staiano AE, Calvert SL. The promise of exergames as tools to measure physical health. Entertain Comput 2011 Jan 01;2(1):17-21 [FREE Full text] [doi: 10.1016/j.entcom.2011.03.008] [Medline: 23378860]

\author{
Abbreviations \\ BMI: body mass index \\ EE: energy expenditure \\ INSPQ: Institut national de santé publique du Québec \\ IPAQ: International Physical Activity Questionnaire \\ MVPA: moderate-to-vigorous physical activity \\ PA: physical activity \\ SDT: self-determination theory
}

\author{
Edited by G Eysenbach; submitted 08.01.19; peer-reviewed by A Staiano, L Gutierrez-Puertas; comments to author 03.02.19; revised \\ version received 31.03.19; accepted 03.04.19; published 31.07.19 \\ Please cite as: \\ O'Loughlin EK, Barnett TA, McGrath JJ, Consalvo M, Kakinami L \\ Factors Associated with Sustained Exergaming: Longitudinal Investigation \\ JMIR Serious Games 2019;7(3):e13335 \\ URL: https://games.jmir.org/2019/3/e13335/ \\ doi: $10.2196 / 13335$ \\ PMID: 31368440
}

CErin Kathleen O'Loughlin, Tracie A Barnett, Jennifer J McGrath, Mia Consalvo, Lisa Kakinami. Originally published in JMIR Serious Games (http://games.jmir.org), 31.07.2019. This is an open-access article distributed under the terms of the Creative Commons Attribution License (https://creativecommons.org/licenses/by/4.0/), which permits unrestricted use, distribution, and reproduction in any medium, provided the original work, first published in JMIR Serious Games, is properly cited. The complete bibliographic information, a link to the original publication on http://games.jmir.org, as well as this copyright and license information must be included. 